\title{
SOLVING DEFICIENT POLYNOMIAL SYSTEMS WITH HOMOTOPIES WHICH KEEP THE SUBSCHEMES AT INFINITY INVARIANT
}

\author{
T. Y. LI AND XIAOSHEN WANG
}

\begin{abstract}
By a deficient polynomial system of $n$ polynomial equations in $n$ unknowns we mean a system that has fewer solutions than that predicted by the total degree, or the Bézout number, of the system. If the system is $m$ homogeneous, the Bézout number can be considerably reduced. In this paper, we introduce a homotopy for numerically determining all isolated solutions of deficient $m$-homogeneous systems. The initial polynomial system $Q$ is chosen which keeps the subschemes of $H(x, t)=(1-t) a Q(x)+t P(x)$ at infinity invariant when $t$ varies in $[0,1)$.
\end{abstract}

\section{INTRODUCTION}

Let $P(x)=0$ denote a system of $n$ polynomial equations in $n$ unknowns. Denoting $P=\left(p_{1}, \ldots, p_{n}\right)$, we want to find all solutions to

$$
p_{1}\left(x_{1}, \ldots, x_{n}\right)=0, \ldots, p_{n}\left(x_{1}, \ldots, x_{n}\right)=0 \text {, }
$$

for $x=\left(x_{1}, \ldots, x_{n}\right) \in \mathbf{C}^{n}$. The homotopy continuation method for solving this system is to define a trivial system

$$
Q(x)=0
$$

and then to follow the curves in the real variable $t$ which make up the solution set of

$$
0=H(x, t)=(1-t) Q(x)+t P(x) .
$$

More precisely, if $Q(x)=0$ is chosen correctly, the following three properties hold:

1. (Triviality) The solutions of $Q(x)=0$ are known.

2. (Smoothness) The solution set of $H(x, t)=0$ for $0 \leq t<1$ consists of a finite number of smooth paths, each parametrized by $t$ in $[0,1)$.

3. (Accessibility) Every isolated solution of $H(x, 1)=P(x)=0$ is reached by some path originating at $t=0$. It follows that this path starts at a solution of $H(x, 0)=Q(x)=0$.

Received August 7, 1989; revised May 11, 1990.

1980 Mathematics Subject Classification (1985 Revision). Primary 65H10, 65H15.

The first author's research was supported in part by NSF under Grant DMS-8902663. 
When these three properties hold, the solution paths can be followed from the initial points (known because of property 1) at $t=0$ to all solutions of the original problem $P(x)=0$ at $t=1$, using standard numerical techniques [1].

It is important to realize that even though properties $1-3$ imply that each solution of $P(x)=0$ will lie at the end of a solution path, it is also consistent with these properties that some of the paths may diverge to infinity as the parameter $t$ approaches 1 . (The smoothness property rules this out for $t \rightarrow$ $t_{0}<1$.)

This method has the virtue of locating all isolated solutions of the system $P(x)=0$. A typical choice of $Q$ that satisfies the three properties $[9,14,17]$ is

$$
q_{1}(x)=c \prod_{i=1}^{d_{1}}\left(x_{1}-\alpha_{1 i}\right), \ldots, q_{n}(x)=c \prod_{i=1}^{d_{n}}\left(x_{n}-\alpha_{n i}\right),
$$

where $d_{1}, \ldots, d_{n}$ are the degrees of $p_{1}(x), \ldots, p_{n}(x)$, and $c, \alpha_{j i}$ are random complex numbers where $c \neq 0$ and $\alpha_{j i}$ are distinct. In this case, the number of paths which need to be followed to arrive at all solutions of $P(x)=0$ is the product $d \equiv d_{1} \cdots d_{n}$. This number, often called the Bézout number, or total degree, of the system, is a classical upper bound on the number of isolated solutions, counting multiplicities. However, in most practical cases we have seen, the number of solutions of (1.1) can turn out to be smaller than $d$, and in some cases only a small fraction of $d$. Such systems are called deficient. When applying homotopy continuation methods to a deficient system, sending out $d$ paths in search of solutions, the paths which do not converge to solutions of (1.1) will go to infinity, representing wasted computation.

For deficient systems, various homotopies have been introduced $[10,11,12$, $15,16]$. In [15], the $m$-homogeneous structure of $(1.1)$, when it is available, is used to reduce the Bézout number and hence the number of paths needed to be followed. Given a polynomial $p$ of degree $d$ in the $n$ variables $x_{1}, \ldots, x_{n}$, we can define its homogenization

$$
\tilde{p}\left(x_{0}, \ldots, x_{n}\right)=\left(x_{0}\right)^{d} p\left(x_{1} / x_{0}, \ldots, x_{1} / x_{0}\right) .
$$

For the polynomial system $P=\left(p_{1}, \ldots, p_{n}\right)$ we use $\widetilde{P}$ to represent $\left(\tilde{p}_{1}, \ldots\right.$, $\left.\tilde{p}_{n}\right)$. A typical suggestion in [10-12] for deficient polynomial systems is to choose $Q(x)$ so as to share a similar type of deficiency as $P(x)$, with the basic assumption that the zeros of $Q(x)$ at infinity, i.e., the zeros of $\widetilde{Q}(x)$ with $x_{0}=0$, are nonsingular, so that the accessibility is guaranteed with a considerably reduced number of solution paths needed to be followed.

In reading [16], we discovered a flaw in the theorem which formed one of the bases in the article. The authors basically claimed a general result that the nonsingularity of the zeros of $Q(x)$ at infinity can be replaced by the following. Let the common zeros of $\widetilde{P}$ and $\widetilde{Q}$ in (1.3) at infinity be denoted by $S$. If for each $s \in S$ the multiplicity of $s$ as a solution of $\widetilde{Q}(x)=0$ is less than or equal to that of $s$ as a solution of $\widetilde{P}(x)=0$, and all other zeros of $\widetilde{Q}(x)$ are isolated 


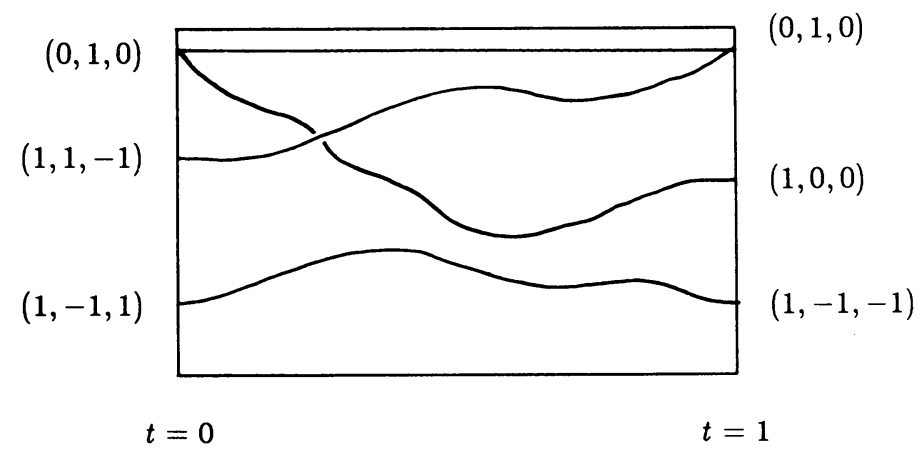

Figure 1

The four solution paths

and nonsingular, then for generic $a \in \mathbf{C}$, by following the solution paths of

$$
\widetilde{H}(x, t)=a(1-t) \widetilde{Q}(x)+t \widetilde{P}(x)=0,
$$

starting from the isolated zeros of $\widetilde{Q}(x)$ outside $S$, one can obtain all isolated zeros of $\widetilde{P}(x)=0$ outside $S$. This assertion can be shown to be in error, as the following example indicates.

Example. Let $P=\left(p_{1}, p_{2}\right)$ and $Q=\left(q_{1}, q_{2}\right)$ be defined by

$$
\begin{aligned}
& p_{1}\left(x_{1}, x_{2}\right)=x_{2}^{2}+x_{1}, \quad p_{2}\left(x_{1}, x_{2}\right)=x_{2}^{2}+x_{2}, \\
& q_{1}\left(x_{1}, x_{2}\right)=x_{2}^{2}-1, \quad q_{2}\left(x_{1}, x_{2}\right)=x_{2}^{2}+x_{1} x_{2} .
\end{aligned}
$$

The common solution set of $\widetilde{P}\left(x_{0}, x_{1}, x_{2}\right)=0$ and $\widetilde{Q}\left(x_{0}, x_{1}, x_{2}\right)=0$ at infinity is $(0,1,0)$ with multiplicity 2 . However, for any nonzero $a \in \mathbf{C}$ which is not a negative real number, by following the solution paths of (1.4) starting from the two zeros of $\widetilde{Q}$ in affine space $(1,-1,1)$ and $(1,1,-1)$, one can only find one of the isolated zeros of $\widetilde{P}(x),(1,-1,-1)$, in affine space. For $a=.59032965+.15799344 i$ the computed results are shown in Figure 1. The solution path starting with $(1,-1,1)$ can reach $(1,-1,-1)$ and the solution path starting from $(1,1,-1)$ goes to infinity as $t$ tends to 1 . A theoretical proof of this assertion for general $a$ is given in the Appendix.

In view of this counterexample, we suggest in this paper an alternative which guarantees the accessibility of the homotopy for deficient polynomial systems. In our homotopy, we choose $\widetilde{Q}(x)$ in such a way that its subscheme at infinity contains the subscheme of $\widetilde{P}(x)$ at infinity. Then, for generic $a \in \mathbf{C}$, the subschemes of $\widetilde{H}(x, t)$ in (1.4) at infinity remain the same for all $t \in[0,1)$. Consequently, solution paths of (1.4) originated at zeros of $\widetilde{Q}(x)$ in affine space stay in affine space for all $t \in[0,1)$. As a result, the typical assumption of nonsingularity of $Q(x)$ at infinity in [10-12] can be dropped.

Our main results are stated in $\S 2$ and proved in $\S 3$ for general $m$-homogeneous deficient polynomial systems. When $m=1$ the conditions given in Theorem 2.1 
and Proposition 2.1 are equivalent to condition (2.4) of Theorem 2.3 in [12]. However, our condition is much easier to verify. In $\S 2$, we also give several examples to which our main results apply.

\section{THEOREMS AND APPLICATIONS}

The complex $n$-space $\mathbf{C}^{n}$ can be naturally embedded in complex projective space

$$
\mathbf{P}^{n}=\left\{\left(x_{0}, \ldots, x_{n}\right) \in \mathbf{C}^{n+1} \backslash(0, \ldots, 0)\right\} / \sim,
$$

where the equivalent relation " $\sim$ " is given by $x \sim y$ if $x=c y$ for some nonzero $c \in \mathbf{C}$. Similarly, the space $N=\mathbf{C}^{k_{1}} \times \cdots \times \mathbf{C}^{k_{m}}$ can be naturally embedded in $M=\mathbf{P}^{k_{1}} \times \cdots \times \mathbf{P}^{k_{m}}$. A point $\left(y_{1}, \ldots, y_{m}\right)$ in $N$ with $y_{i}=\left(y_{1}^{i}, \ldots, y_{k_{i}}^{i}\right), i=$ $1, \ldots, m$, corresponds to a point $\left(z_{1}, \ldots, z_{m}\right)$ in $M$ with $z_{i}=\left(z_{0}^{i}, \ldots, z_{k_{i}}^{i}\right)$ and $z_{0}^{i}=1, i=1, \ldots, m$. The set of such points in $M$ is usually called the affine space in this setting. The points in $M$ with at least one $z_{0}^{i}=0$ are called the points at infinity.

Given a polynomial $p$ in the $n$ variables $x_{1}, \ldots, x_{n}$, if we divide the variables into $m$ groups $y_{1}=\left(x_{1}^{1}, \ldots, x_{k_{1}}^{1}\right), y_{2}=\left(x_{1}^{2}, \ldots, x_{k_{2}}^{2}\right), \ldots, y_{m}=$ $\left(x_{1}^{m}, \ldots, x_{k_{m}}^{m}\right)$ with $k_{1}+\cdots+k_{m}=n$ and let $d_{i}$ be the degree of $p$ with respect to $y_{i}$, then we can define its $m$-homogenization as

$$
\tilde{p}\left(z_{1}, \ldots, z_{m}\right)=\left(z_{0}^{1}\right)^{d_{1}} \times \cdots \times\left(z_{0}^{m}\right)^{d_{m}} p\left(y_{1} / z_{0}^{1}, \ldots, y_{m} / z_{0}^{m}\right),
$$

which is homogeneous with respect to each $z_{i}=\left(z_{0}^{i}, \ldots, z_{k_{i}}^{i}\right) \in \mathbf{P}^{k_{i}}, i=$ $1, \ldots, m$. Here, $z_{j}^{i}=x_{j}^{i}$, for $j \neq 0$. Such a polynomial is said to be $m$ homogeneous. To illustrate this definition, let us consider the polynomial

$$
\begin{aligned}
p\left(\lambda, x_{1}, \ldots, x_{n}\right)= & \lambda^{2}\left(a_{1} x_{1}+\cdots+a_{n} x_{n}-a\right) \\
& +\lambda\left(b_{1} x_{1}+\cdots+b_{n} x_{n}-b\right)+\left(c_{1} x_{1}+\cdots+c_{n} x_{n}-c\right) .
\end{aligned}
$$

We may let $y_{1}=(\lambda), y_{2}=\left(x_{1}, \ldots, x_{n}\right)$ and $z_{1}=\left(\lambda_{0}, \lambda\right), z_{2}=\left(x_{0}, x_{1}, \ldots\right.$, $x_{n}$ ). The degree of $p$ is 2 with respect to $y_{1}$ and 1 with respect to $y_{2}$. Hence, its 2-homogenization is

$$
\begin{aligned}
\tilde{p}\left(\lambda_{0}, \lambda, x_{0}, x_{1}, \ldots, x_{n}\right)= & \lambda^{2}\left(a_{1} x_{1}+\cdots+a_{n} x_{n}-a x_{0}\right) \\
& +\lambda \lambda_{0}\left(b_{1} x_{1}+\cdots+b_{n} x_{n}-b x_{0}\right) \\
& +\lambda_{0}^{2}\left(c_{1} x_{1}+\cdots+c_{n} x_{n}-c x_{0}\right),
\end{aligned}
$$

which is homogeneous with respect to $\left(\lambda_{0}, \lambda\right)$ and $\left(x_{0}, x_{1}, \ldots, x_{n}\right)$.

For $z_{i}=\left(z_{0}^{i}, \ldots, z_{k_{i}}^{i}\right) \in \mathbf{P}^{k_{i}}, i=1, \ldots, m$, let $S=\mathbf{C}\left[z_{0}^{1}, z_{1}^{1}, \ldots, z_{k_{m}}^{m}\right]$ be the ring of polynomials in the variables $z_{i}$ with complex coefficients. If ${ }^{m} A$ is an ideal and $T$ is a prime ideal of $S$, then denote by $A^{T}$ the ideal

$$
A^{T}=\{f \in S \mid h f \in A \text { for some } h \notin T\} .
$$


For a point $z=\left(z_{1}, \ldots, z_{m}\right) \in \mathbf{P}^{k_{1}} \times \cdots \times \mathbf{P}^{k_{m}}$, let $I_{z}$ denote the maximal ideal $\{f \in S \mid f(z)=0\}$. If $f_{1}, \ldots, f_{n}$ are $m$-homogeneous polynomials in the variables $\left(z_{1}, \ldots, z_{m}\right) \in P^{k_{1}} \times \cdots \times P^{k_{m}}$, we denote by $V\left(f_{1}, \ldots, f_{r}\right)$ the common zero set of $f_{1}, \ldots, f_{r}$ in $\mathbf{P}^{k_{1}} \times \cdots \times \mathbf{P}^{k_{m}}$. We say a point $y \in$ $V\left(f_{1}, \ldots, f_{r}\right)$ is nonsingular if

$$
\operatorname{rank} \frac{\partial\left(f_{1}, \ldots, f_{r}\right)}{\partial\left(z_{1}, \ldots, z_{m}\right)}=\operatorname{codim}_{y}\left(V\left(f_{1}, \ldots, f_{r}\right), P^{k_{1}} \times \cdots \times P^{k_{m}}\right),
$$

where codim denotes complex codimension. We denote by $\left\langle f_{1}, \ldots, f_{r}\right\rangle$ the ideal generated by $f_{1}, \ldots, f_{r}$. To be more precise, $\left\langle f_{1}, \ldots, f_{r}\right\rangle$ is the set of all polynomials of the form

$$
\sum_{i=1}^{r} g_{i} f_{i}
$$

where the $g_{i}$ 's are polynomials in $S$.

Given the system $P(x)=\left(p_{1}(x), \ldots, p_{n}(x)\right)$ in $(1.1)$, let $Q(x)=\left(q_{1}(x)\right.$, $\left.\ldots, q_{n}(x)\right)$ and

$$
H(a, x, t)=(1-t) a Q(x)+t P(x), \quad a \in \mathbf{C} .
$$

Here, we consider $x \in \mathbf{C}^{k_{1}} \times \mathbf{C}^{k_{2}} \times \cdots \times \mathbf{C}^{k_{m}}$ with $k_{1}+k_{2}+\cdots+k_{m}=n, t$ real and $\operatorname{deg} p_{i}=\operatorname{deg} q_{i}, i=1, \ldots, n$. Let

$$
\begin{aligned}
& \widetilde{H}(a, z, t)=(1-t) a \widetilde{Q}(z)+t \widetilde{P}(z), \\
& \qquad t \in[0,1], \quad z \in M=P^{k_{1}} \times \cdots \times P^{k_{m}},
\end{aligned}
$$

which is the $m$-homogenization of $(2.1)$. Let $\langle\widetilde{Q}\rangle=\left\langle\tilde{q}_{1}, \ldots, \tilde{q}_{n}\right\rangle$ and $\langle\widetilde{P}\rangle=$ $\left\langle\tilde{p}_{1}, \ldots, \tilde{p}_{n}\right\rangle$. Our main results are the following.

Theorem 2.1. Suppose that the polynomial system $\widetilde{Q}$ in (2.2) has the following properties:

(1) for every point $z$ at infinity, $\langle\widetilde{Q}\rangle^{I_{z}} \supseteq\langle\widetilde{P}\rangle^{I_{z}}$;

(2) the set $T=\left\{\right.$ the points of $V\left(\tilde{q}_{1}, \ldots, \tilde{q}_{n}\right)$ in affine space $\}$ consists of nonsingular isolated points $x^{1}, \ldots, x^{r}$.

Then there exists an open dense subset $D$ of $\mathbf{C}$ with full measure, such that for $a^{-1}$ chosen from $D$, we have

(a) (Smoothness) For each isolated zero $x^{k} \in T, k=1, \ldots, r$, there is a function $x^{k}(t):[0,1] \rightarrow M$ which is analytic and contained in affine space for all $t$ in $[0,1)$ and satisfies $\widetilde{H}\left(a, x^{k}(t), t\right)=0$.

(b) (Accessibility) Each isolated solution of $P(x)=0$ is reached by $x^{k}(t)$ for some $k$ at $t=1$.

Remark 2.1. If $z \notin V\left(\tilde{p}_{1}, \ldots, \tilde{p}_{n}\right)$, then there exists an $h \in\left\langle\tilde{p}_{1}, \ldots, \tilde{p}_{n}\right\rangle$ such that $h(z) \neq 0$, i.e., $h \notin I_{z}$. Thus, $\langle\widetilde{P}\rangle^{I_{z}}=\{f \in S \mid f h \in\langle\widetilde{P}\rangle$ for some $\left.h \notin I_{z}\right\}=S$. Hence, condition (1) above implies that every point 
of $V\left(\tilde{q}_{1}, \ldots, \tilde{q}_{n}\right)$ at infinity is also a point of $V\left(\tilde{p}_{1}, \ldots, \tilde{p}_{n}\right)$. By the same argument, if $z \notin V\left(\tilde{q}_{1}, \ldots, \tilde{q}_{n}\right)$, then $\langle\tilde{Q}\rangle^{I_{z}}=S$, and (1) is obvious. So, in order to check condition (1) of Theorem 2.1 one only needs to check this condition for those points at infinity which lie in $V\left(\tilde{q}_{1}, \ldots, \tilde{q}_{n}\right)$. Consequently, condition (1) implies that the subscheme at infinity of the polynomial system $\widetilde{Q}(z)$ contains the subscheme of $\widetilde{P}(z)$ at infinity. (For general definitions and properties of scheme and subscheme, see [7, pp. 60-190].)

Remark 2.2. By a straightforward verification one can easily see that $\left\langle\langle\widetilde{Q}\rangle^{I_{z}}\right\rangle^{I_{z}}=$ $\langle\widetilde{Q}\rangle^{I_{z}}$. Hence, if $\langle\widetilde{Q}\rangle^{I_{z}} \supseteq\langle\widetilde{P}\rangle$, then $\langle\widetilde{Q}\rangle^{I_{z}} \supseteq\langle\widetilde{P}\rangle^{I_{z}}$.

Remark 2.3. In the counterexample (1.5), (1.6) of $\S 1$,

$$
\begin{array}{cc}
\tilde{p}_{1}\left(x_{0}, x_{1}, x_{2}\right)=x_{2}^{2}+x_{1} x_{0}, & \tilde{p}_{2}\left(x_{0}, x_{1}, x_{2}\right)=x_{2}^{2}+x_{2} x_{0}, \\
\tilde{q}_{1}\left(x_{0}, x_{1}, x_{2}\right)=x_{2}^{2}-x_{0}^{2}, & \tilde{q}_{2}\left(x_{0}, x_{1}, x_{2}\right)=x_{2}^{2}+x_{1} x_{2} .
\end{array}
$$

At $z=\left(x_{0}, x_{1}, x_{2}\right)=(0,1,0),\langle\widetilde{Q}\rangle^{I_{z}} \nsupseteq\langle\widetilde{P}\rangle^{I_{z}}$. This can be shown as follows. Since $\tilde{p}_{2}-\tilde{p}_{1}=x_{0}\left(x_{2}-x_{1}\right) \in\langle\widetilde{P}\rangle^{I_{z}}$ and $x_{2}-x_{1} \neq 0$ at $z=(0,1,0)$, we have $x_{0} \in\langle\widetilde{P}\rangle^{I_{z}}$. Thus, $x_{2}^{2} \in\langle\widetilde{P}\rangle^{I_{z}}$. So $\langle\widetilde{P}\rangle^{I_{z}}=\left\langle x_{0}, x_{2}^{2}\right\rangle$. Since $\left(\tilde{q}_{2}-\tilde{q}_{1}\right) x_{2}-\tilde{q}_{1} x_{1}=$ $x_{0}^{2}\left(x_{1}+x_{2}\right) \in\langle\widetilde{Q}\rangle^{I_{z}}$ and $x_{1}+x_{2} \neq 0$ at $z$, we have $x_{0}^{2} \in\langle\widetilde{Q}\rangle^{I_{z}}$. Therefore, $\langle\widetilde{Q}\rangle^{I_{z}}=\left\langle x_{2}, x_{0}^{2}\right\rangle$. Evidently, $\langle\widetilde{Q}\rangle^{I_{z}} \nsupseteq\langle\widetilde{P}\rangle^{I_{z}}$.

The following proposition indicates that Theorem 2.1 is a generalization of the main result in [10].

Proposition 2.1. Suppose that the polynomial systems $\widetilde{Q}, \widetilde{P}$ in (2.2) have the following properties:

(1) every point of $V(\widetilde{Q})$ at infinity is also a point of $V(\widetilde{P})$;

(2) the set $T=\{$ the set of points of $V(\tilde{Q})$ in affine space $\}$ consists of nonsingular isolated points.

Then for a nonsingular point $z$ of $V(\widetilde{Q})$ at infinity, $\langle\widetilde{Q}\rangle^{I_{z}} \supseteq\langle\widetilde{P}\rangle^{I_{z}}$.

Example 2.1. Suppose we want to solve the system

$$
p_{1}(x, y)=x y+y+1=0, \quad p_{2}(x, y)=x^{3} y^{2}-x y+1=0 .
$$

By considering $(x, y) \in C^{1} \times C^{1}$, we may 2-homogenize (2.3) as

$$
\begin{aligned}
& \tilde{p}_{1}\left(x_{0}, x, y_{0}, y\right)=x y+x_{0} y+x_{0} y_{0}=0, \\
& \tilde{p}_{2}\left(x_{0}, x, y_{0}, y\right)=x^{3} y^{2}-x x_{0}^{2} y y_{0}+x_{0}^{3} y_{0}^{2}=0,
\end{aligned}
$$

where $\left(x_{0}, x, y_{0}, y\right) \in \mathbf{P}^{1} \times \mathbf{P}^{1}$. Then this system $\widetilde{P}=\left(\tilde{p}_{1}, \tilde{p}_{2}\right)$ has one solution $(0,1,1,0)$ at infinity with multiplicity 2 , and three affine solutions. Our starting system $Q=\left(q_{1}, q_{2}\right)$ can be chosen as

$$
q_{1}(x, y)=x y+y+1=0, \quad q_{2}(x, y)=x^{3} y^{2}-x y=0 .
$$


Its 2-homogenization is

$$
\begin{aligned}
& \tilde{q}_{1}\left(x_{0}, x, y_{0}, y\right)=x y+x_{0} y+x_{0} y_{0}=0, \\
& \tilde{q}_{2}\left(x_{0}, x, y_{0}, y\right)=x^{3} y^{2}-x x_{0}^{2} y y_{0}=0 .
\end{aligned}
$$

The system $Q$ has three nonsingular solutions $(x, y)=(0,-1),(-1 / 2+$ $\sqrt{3} i / 2,-1 / 2+\sqrt{3} i / 2)$ and $(-1 / 2-\sqrt{3} i / 2,-1 / 2-\sqrt{3} i / 2)$, and its solution at infinity is the same as that of $P$. Write $\tilde{q}_{2}=x g$ with $g=x^{2} y^{2}-x_{0}^{2} y_{0} y$. Since $x \neq 0$ at $z=(0,1,1,0)$, we have $x \notin I_{z}$, so, $g \in\langle\widetilde{Q}\rangle^{I_{z}}$. Further, $\tilde{q}_{1} x y-g=x_{0} y\left(x_{0} y_{0}+x y+x y_{0}\right) \in\langle\widetilde{Q}\rangle^{I_{z}}$ and $x_{0} y_{0}+x y+x y_{0} \neq 0$ at $z$ imply $x_{0} y \in\langle\widetilde{Q}\rangle^{I_{z}}$. Since $x_{0} y_{0} \tilde{q}_{1} \in\langle\widetilde{Q}\rangle^{I_{z}}$, we have $x_{0}^{2} y_{0}^{2}=x_{0} y_{0} \tilde{q}_{1}-x_{0} y\left(x y_{0}+x_{0} y_{0}\right) \epsilon$ $\langle\widetilde{Q}\rangle^{I_{z}}$ and thus $\tilde{p}_{2}=\tilde{q}_{2}+x_{0}\left(x_{0}^{2} y_{0}^{2}\right) \in\langle\widetilde{Q}\rangle^{I_{z}}$. Along with $\tilde{p}_{1}=\tilde{q}_{1} \in\langle\widetilde{Q}\rangle^{I_{z}}$, we have $\langle\widetilde{Q}\rangle^{I_{z}} \supseteq\langle\widetilde{P}\rangle^{I_{z}}$. So Theorem 2.1 applies. It provides a homotopy and three paths, beginning from the roots of $(2.5)$, which lead to all roots of (2.3).

Table 2.1 shows the computed results.

TABLE 2.1

\begin{tabular}{|c|c|c|c|c|}
\hline \multicolumn{5}{|c|}{ Parameter $a=-.13960695-.6281187 i$} \\
\hline \multicolumn{3}{|c|}{ Starting Point } & \multicolumn{2}{|c|}{ Solution Reached } \\
\hline & $x$ & $y$ & $x$ & $y$ \\
\hline 1. & 0 & -1 & -.4301591 & -1.7548765 \\
\hline 2. & $-1 / 2+\sqrt{3} i / 2$ & $-1 / 2+\sqrt{3} i / 2$ & $-.78492+1.307138 i$ & $-.1225614+.7448609 i$ \\
\hline 3. & $-1 / 2-\sqrt{3} i / 2$ & $-1 / 2-\sqrt{3} i / 2$ & $-.78492-1.3071413 i$ & $-.1225611-.7448618 i$ \\
\hline
\end{tabular}

Solutions to $(2.3)$

The notion of $m$-homogeneous, when $m=1$, is the same as homogeneous. For homogeneous polynomials $f_{1}, \ldots, f_{r}$ we use $\left\langle f_{1}, \ldots, f_{r}\right\rangle_{e}$ to denote the subset of $\left\langle f_{1}, \ldots, f_{r}\right\rangle$ consisting of homogeneous polynomials of degree $e$. In [12], the following condition on $\widetilde{P}, \widetilde{Q}$ in $(2.2)$ is used to guarantee the accessibility of the "random product homotopy" paths: For each positive integer $k$,

$$
\left\langle\tilde{q}_{1}, \ldots, \tilde{q}_{n}, x_{0}^{k}\right\rangle_{e} \supseteq\left\langle\tilde{p}_{1}, \ldots, \tilde{p}_{n}, x_{0}^{k}\right\rangle_{e}
$$

for all sufficiently large $e$.

The following proposition shows that condition (2.6) is equivalent to condition (1) in Theorem 2.1 when $m=1$. However, we shall illustrate in Example 2.2 that condition (1) in Theorem 2.1 can be much easier to verify.

Proposition 2.2. Suppose that the polynomial systems $\widetilde{P}$ and $\widetilde{Q}$ in (2.2) are homogeneous. Then for every point $z$ at infinity, $\langle\widetilde{Q}\rangle^{I_{z}} \supseteq\langle\widetilde{P}\rangle^{I_{z}}$ if and only if for each positive integer $k$,

$$
\left\langle\tilde{q}_{1}, \ldots, \tilde{q}_{n}, x_{0}^{k}\right\rangle_{e} \supseteq\left\langle\tilde{p}_{1}, \ldots, \tilde{p}_{n}, x_{0}^{k}\right\rangle_{e}
$$

for all sufficiently large $e$. 
Example 2.2. The following system is the mathematical model of a lumpedparameter chemically reacting system [2]:

$$
\begin{aligned}
& p_{1}\left(x_{1}, x_{2}, x_{3}, x_{4}\right)=-a_{1} x_{1}\left(1-x_{3}-x_{4}\right)+a_{2} x_{3}-\left(x_{1}-b_{1}\right), \\
& p_{2}\left(x_{1}, x_{2}, x_{3}, x_{4}\right)=-a_{3} x_{2}\left(1-x_{3}-x_{4}\right)+a_{4} x_{4}-\left(x_{2}-b_{2}\right), \\
& p_{3}\left(x_{1}, x_{2}, x_{3}, x_{4}\right)=a_{1} x_{1}\left(1-x_{3}-x_{4}\right)-a_{2} x_{3}-a_{5} x_{3} x_{4}, \\
& p_{4}\left(x_{1}, x_{2}, x_{3}, x_{4}\right)=a_{3} x_{2}\left(1-x_{3}-x_{4}\right)-a_{4} x_{4}-a_{5} x_{3} x_{4} .
\end{aligned}
$$

While the Bézout number of the system $P=\left(p_{1}, p_{2}, p_{3}, p_{4}\right)$ is 16 , for generic $a_{i}$ 's and $b_{i}$ 's there are only four zeros of (2.7) [2]. Define $Q=\left(q_{1}, q_{2}, q_{3}, q_{4}\right)$ by

$$
\begin{aligned}
& q_{1}\left(x_{1}, x_{2}, x_{3}, x_{4}\right)=\left(x_{1}-1\right)\left(1-x_{3}-x_{4}\right), \\
& q_{2}\left(x_{1}, x_{2}, x_{3}, x_{4}\right)=\left(x_{2}-i\right)\left(2-x_{3}-x_{4}\right), \\
& q_{3}\left(x_{1}, x_{2}, x_{3}, x_{4}\right)=x_{1}\left(2-x_{3}-x_{4}\right)+x_{3} x_{4}, \\
& q_{4}\left(x_{1}, x_{2}, x_{3}, x_{4}\right)=\left(x_{2}+1\right)\left(1-x_{3}-x_{4}\right)+x_{3} x_{4} .
\end{aligned}
$$

The homogenization $\widetilde{Q}$ of $Q$ is

$$
\begin{aligned}
& \tilde{q}_{1}\left(x_{0}, x_{1}, x_{2}, x_{3}, x_{4}\right)=\left(x_{1}-x_{0}\right)\left(x_{0}-x_{3}-x_{4}\right), \\
& \tilde{q}_{2}\left(x_{0}, x_{1}, x_{2}, x_{3}, x_{4}\right)=\left(x_{2}-i x_{0}\right)\left(2 x_{0}-x_{3}-x_{4}\right), \\
& \tilde{q}_{3}\left(x_{0}, x_{1}, x_{2}, x_{3}, x_{4}\right)=x_{1}\left(2 x_{0}-x_{3}-x_{4}\right)+x_{3} x_{4}, \\
& \tilde{q}_{4}\left(x_{0}, x_{1}, x_{2}, x_{3}, x_{4}\right)=\left(x_{2}+x_{0}\right)\left(x_{0}-x_{3}-x_{4}\right)+x_{3} x_{4} .
\end{aligned}
$$

The points of $V(\tilde{Q})$ at infinity are $\left(x_{0}, x_{1}, x_{2}, x_{3}, x_{4}\right)=(0,0,0,0,1)$, $(0,0,0,1,0)$ and a line $I=\left(0, x_{1}, x_{2}, 0,0\right)$. The rank of

$$
\left.\frac{\partial\left(\tilde{q}_{1}, \tilde{q}_{2}, \tilde{q}_{3}, \tilde{q}_{4}\right)}{\partial\left(x_{0}, x_{1}, x_{2}, x_{3}, x_{4}\right)}\right|_{(0,0,0,0,1)}=\left[\begin{array}{rrrrr}
1 & -1 & 0 & 0 & 0 \\
i & 0 & -1 & 0 & 0 \\
0 & -1 & 0 & 1 & 0 \\
-1 & 0 & -1 & 1 & 0
\end{array}\right]
$$

is 4 and hence, $(0,0,0,0,1)$ is nonsingular; similarly for $(0,0,0,1,0)$. These two points also belong to $V(\widetilde{P})$. Further, the system $(2.8)$ has four nonsingular isolated zeros: $\left(x_{1}, x_{2}, x_{3}, x_{4}\right)=(1,-1,0,2),(1,-1,2,0)$, $(0, i, 0,1)$, and $(0, i, 1,0)$. So, Proposition 2.1 applies. That is,

$$
\left\langle\tilde{q}_{1}, \tilde{q}_{2}, \tilde{q}_{3}, \tilde{q}_{4}\right\rangle^{I_{z}} \supseteq\left\langle\tilde{p}_{1}, \tilde{p}_{2}, \tilde{p}_{3}, \tilde{p}_{4}\right\rangle^{I_{z}}
$$

for $z=(0,0,0,0,1),(0,0,0,1,0)$. For $z \in I=\left(0, x_{1}, x_{2}, 0,0\right)$, either $x_{1} \neq 0$ or $x_{2} \neq 0$, say $x_{1} \neq 0$. Then $x_{1}-x_{0} \neq 0$ at $z$. So, from $\tilde{q}_{1}$,

$$
x_{0}-x_{3}-x_{4} \in\langle\tilde{Q}\rangle^{I_{2}} \text {. }
$$

It follows from $\tilde{q}_{4}$, that $x_{3} x_{4} \in\langle\widetilde{Q}\rangle^{I_{z}}$ and hence $x_{1}\left(2 x_{0}-x_{3}-x_{4}\right) \in\langle\widetilde{Q}\rangle^{I_{z}}$, from $\tilde{q}_{3}$. Since $x_{1} \neq 0$ at $z$, we have $2 x_{0}-x_{3}-x_{4} \in\langle\widetilde{Q}\rangle^{I_{2}}$. Comparing with (2.10) yields $x_{0} \in\langle\widetilde{Q}\rangle^{I_{z}}$. Accordingly, it is easy to see that $\langle\widetilde{Q}\rangle^{I_{z}} \supseteq\langle\widetilde{P}\rangle$. Thus 
by Remark 2.2, we have $\langle\widetilde{Q}\rangle^{I_{z}} \supseteq\langle\widetilde{P}\rangle^{I_{z}}$. So Theorem 2.1 provides a homotopy and four paths which lead to all roots of (2.7).

Table 2.2 shows the computed result.

TABLE 2.2

Zeros of (2.7) in $C^{4}$

\begin{tabular}{|l|l|}
\hline \multicolumn{2}{|c|}{ Parameters } \\
\hline$a_{1}=.76771879+.32820278 i$ & $a_{5}=.89248163+.45296562 i$ \\
\hline$a_{2}=.54890949+.1093949 i$ & $b_{1}=.04796080+.88868678 i$ \\
\hline$a_{3}=.33010021+.89058417 i$ & $b_{2}=.82915151+.66987747 i$ \\
\hline$a_{4}=.11129092+.67177492 i$ & $a=.59527814+.71154547 i$ \\
\hline
\end{tabular}

\begin{tabular}{|l|l|l|l|l|}
\hline \multicolumn{5}{|c|}{ Starting Points } \\
\hline 1. & $x_{1}=1$ & $x_{2}=-1$ & $x_{1}=-.731938+.3453089 i$ & $x_{2}=.049258+.1264814 i$ \\
\hline & $x_{3}=0$ & $x_{4}=2$ & $x_{3}=2.9605355+7.79467 i$ & $x_{4}=.0547949-.0998576 i$ \\
\hline 2. & $x_{1}=1$ & $x_{2}=-1$ & $x_{1}=-2.214090+1.074372 i$ & $x_{2}=-1.43290+.8555617 i$ \\
\hline & $x_{3}=2$ & $x_{4}=0$ & $x_{3}=.6873539-1.0286067 i$ & $x_{4}=1.6660806+.7643948 i$ \\
\hline 3. & $x_{1}=0$ & $x_{2}=i$ & $x_{1}=0.433732+.750366 i$ & $x_{2}=.8245659+.5315566 i$ \\
\hline & $x_{3}=0$ & $x_{4}=1$ & $x_{3}=.1027011+.2787743 i$ & $x_{4}=.4602318-.0694782 i$ \\
\hline 4. & $x_{1}=0$ & $x_{2}=i$ & $x_{1}=.8908184-.8041871 i$ & $x_{2}=1.6720086-1.0229946 i$ \\
\hline & $x_{3}=1$ & $x_{2}=0$ & $x_{3}=1.6573049-1.607046 i$ & $x_{4}=-.5652349+.591969 i$ \\
\hline
\end{tabular}

Example 2.3. For generalized eigenvalue problems (or $\lambda$-matrix problems), the system $P$ has the following form:

$$
\begin{array}{r}
\lambda^{k} B_{0} x+\lambda^{k-1} B_{1} x+\cdots+B_{k} x=0, \\
1+\alpha_{1} x_{1}+\cdots+\alpha_{n} x_{n}=0,
\end{array}
$$

where $x=\left(x_{1}, \ldots, x_{n}\right), k>1$, and $B_{0}, \ldots, B_{k}$ are $n \times n$ matrices. Consider $\left(\lambda, x_{1}, \ldots, x_{n}\right) \in C^{1} \times C^{n}$. With 2-homogenization, (2.11) becomes

$$
\begin{aligned}
\lambda^{k} B_{0} x+\lambda^{k-1} \lambda_{0} B_{1} x+\cdots+\left(\lambda_{0}\right)^{k} B_{k} x & =0, \\
x_{0}+\alpha_{1} x_{1}+\cdots+\alpha_{n} x_{n} & =0,
\end{aligned}
$$

with $\left(\lambda_{0}, \lambda, x_{0}, \ldots, x_{n}\right) \in \mathbf{P}^{1} \times \mathbf{P}^{n}$. If $B_{0}$ is a nonsingular matrix, it is quite obvious that (2.12) has $k n$ solutions for generic $\alpha_{i}$ 's. In [4], a homotopy is given for nonsingular $B_{0}$, which provides $k n$ paths leading to all roots of (2.11).

Here, we give an example to which Theorem 2.1 can be applied when $B_{0}$ is singular. For $n=3$ and $k=2$, let

$$
B_{0}=\left[\begin{array}{lll}
0 & 1 & 0 \\
0 & 1 & 0 \\
0 & 0 & 1
\end{array}\right], \quad B_{1}=\left[\begin{array}{lll}
0 & 1 & 0 \\
0 & 0 & 1 \\
1 & 0 & 0
\end{array}\right], \quad B_{2}=\left[\begin{array}{lll}
1 & 0 & 0 \\
0 & 0 & 1 \\
0 & 1 & 0
\end{array}\right],
$$


and $\alpha_{1}=\cdots=\alpha_{n}=1$. Then (2.12) becomes

$$
\begin{aligned}
& \tilde{p}_{1}=\lambda^{2} x_{2}+\lambda \lambda_{0} x_{2}+\lambda_{0}^{2} x_{1}=0, \\
& \tilde{p}_{2}=\lambda^{2} x_{2}+\lambda \lambda_{0} x_{3}+\lambda_{0}^{2} x_{3}=0, \\
& \tilde{p}_{3}=\lambda^{2} x_{3}+\lambda \lambda_{0} x_{1}+\lambda_{0}^{2} x_{2}=0, \\
& \tilde{p}_{4}=x_{0}+x_{1}+x_{2}+x_{3}=0,
\end{aligned}
$$

and the solution set at infinity is $v=\left\{\left(\lambda_{0}, \lambda, x_{0}, x_{1}, x_{2}, x_{3}\right) \mid \lambda_{0}=0, \lambda=1\right.$, $\left.x_{2}=0, x_{0}+x_{1}=0, x_{3}=0\right\}$. Define $Q=\left(q_{1}, q_{2}, q_{3}, q_{4}\right)$ by

$$
\begin{aligned}
& q_{1}=(\lambda-1)(\lambda-2) x_{2}=0, \\
& q_{2}=(\lambda-3)(\lambda-4) x_{3}=0, \\
& q_{3}=(\lambda-3)(\lambda-4) x_{3}+\lambda x_{1}=0, \\
& q_{4}=1+x_{1}+x_{2}+x_{3}=0 .
\end{aligned}
$$

It is easy to check that the zero set at infinity of

$$
\begin{aligned}
& \tilde{q}_{1}=\left(\lambda-\lambda_{0}\right)\left(\lambda-2 \lambda_{0}\right) x_{2}=0, \\
& \tilde{q}_{2}=\left(\lambda-3 \lambda_{0}\right)\left(\lambda-4 \lambda_{0}\right) x_{3}=0, \\
& \tilde{q}_{3}=\left(\lambda-3 \lambda_{0}\right)\left(\lambda-4 \lambda_{0}\right) x_{3}+\lambda \lambda_{0} x_{1}=0, \\
& \tilde{q}_{4}=x_{0}+x_{1}+x_{2}+x_{3}=0
\end{aligned}
$$

is the same as that of (2.13). The system (2.14) has five nonsingular solutions $\left(\lambda, x_{1}, x_{2}, x_{3}\right)=(0,-1,0,0),(1,0,-1,0),(2,0,-1,0),(3,0,0,-1)$, and $(4,0,0,-1)$. For any $z \in v$, we have $\lambda_{0}=0$ and $\lambda=1$, hence $\left(\lambda-3 \lambda_{0}\right)\left(\lambda-4 \lambda_{0}\right) \neq 0$ and $\left(\lambda-\lambda_{0}\right)\left(\lambda-2 \lambda_{0}\right) \neq 0$. From $\tilde{q}_{1}$ and $\tilde{q}_{2}$, both $x_{2}$ and $x_{3}$ are in $\langle\widetilde{Q}\rangle^{I_{z}}$, and from $\tilde{q}_{3}, \lambda_{0} x_{1} \in\langle\widetilde{Q}\rangle^{I_{z}}$. In summary, $\langle\widetilde{Q}\rangle^{I_{z}} \supseteq\langle\widetilde{P}\rangle^{I_{z}}$, and Theorem 2.1 applies.

Table 2.3 shows our computed results.

TABLE 2.3

Solutions to (2.13)

\begin{tabular}{|l|c|c|c|c|}
\hline \multicolumn{5}{|c|}{ Parameter $a=-.74127114+.70628309 i$} \\
\hline & $x_{1}$ & $x_{2}$ & $x_{3}$ & $\lambda$ \\
\hline 1. & -4.0795970 & 3.075972 & 0 & .7548779 \\
\hline 2. & $-.4602-.1825814 i$ & $-.5397982+.1825814 i$ & 0 & $-.8774412+.7448597 i$ \\
\hline 3. & $-.4602+.1825814 i$ & $-.5397982-.1825814 i$ & 0 & $-.8774414-.7448597 i$ \\
\hline 4. & -.33333333 & -.333333333 & -.3333333 & $-.5-.866025 i$ \\
\hline 5. & -.33333333 & -.333333333 & -.3333333 & $-.5+.866025 i$ \\
\hline
\end{tabular}

\section{Proofs of THE THEOREMS}

In this section we prove Theorem 2.1, Proposition 2.1, and Proposition 2.2. Some fundamental results in algebraic geometry are necessary. 
We recall the notations introduced in the last section: $M=\mathbf{P}^{k_{1}} \times \cdots \times \mathbf{P}^{k_{m}}$ with $k_{1}+\cdots+k_{m}=n$, and $S=\mathbf{C}\left[z_{1}, \ldots, z_{m}\right]$, the polynomial ring, where $z_{i}=\left[z_{0}^{i}, \ldots, z_{k_{i}}^{i}\right] \in \mathbf{P}^{k_{i}}, i=1, \ldots, m$. Let $R$ be a ring of polynomials (perhaps a quotient of $S$ ) and $q$ a prime ideal of $R$. We denote by $R_{(q)}$ the localization of $R$ at $q$. The local ring $R_{(q)}$ is made up of "formal fractions" $\left\{\frac{f}{g} \mid f \in R, g \notin q, \operatorname{deg} f=\operatorname{deg} g\right.$ with respect to each $\left.z_{i}, i=1, \ldots, m\right\}$ such that $\frac{f_{1}}{g_{1}}=\frac{f_{2}}{g_{2}}$ if and only if $f_{1} g_{2}=f_{2} g_{1}$ in $R$.

Lemma 3.1. Let $A$ and $B$ be two ideals of $S$, and $z \in M$. If $A^{I_{z}}=B^{I_{z}}$, then $A_{\left(I_{z}\right)}=B_{\left(I_{z}\right)}$.

Proof. For $\frac{b}{f} \in B_{\left(I_{z}\right)}$, we have $b \in B$ and $f(z) \neq 0$. Since $A^{I_{z}}=B^{I_{z}} \supseteq B$, there exists $g \in S$ such that $g(z) \neq 0$ and $g b \in A$. Thus, $\frac{g b}{g f} \in A_{\left(I_{z}\right)}$, so, $A_{\left(I_{z}\right)} \supseteq B_{\left(I_{z}\right)}$. By the same argument, $B_{\left(I_{z}\right)} \subseteq A_{\left(I_{z}\right)}$.

Given a system of polynomials $P(x)=\left(p_{1}(x), \ldots, p_{n}(x)\right)$, let $Q(x)=$ $\left(q_{1}(x), \ldots, q_{n}(x)\right)$ with $\operatorname{deg} p_{i}=\operatorname{deg} q_{i}$. Here we consider $x \in \mathbf{C}^{k_{1}} \times \cdots \times \mathbf{C}^{k_{m}}$ with $k_{1}+\cdots+k_{m}=n$. Define the homotopy

$$
H(a, x, t)=(1-t) a Q(x)+t P(x), \quad t \in[0,1], a \in \mathbf{C},
$$

with $m$-homogenization

$$
\widetilde{H}(a, z, t)=(1-t) a \widetilde{Q}(z)+t \widetilde{P}(z), \quad t \in[0,1], a \in \mathbf{C}, \quad z \in M .
$$

Lemma 3.2. If

$$
\langle\widetilde{Q}\rangle^{I_{y}} \supseteq\langle\widetilde{P}\rangle^{I_{y}}
$$

for any point $y$ at infinity, then there exists a subset $D_{1}$ of $\mathbf{C}$,

$$
D_{1}=\left\{r e^{i \theta} \in C \mid \theta \in[0,2 \pi) \backslash F, F \text { a finite set }, r>0\right\},
$$

such that for any $y$ at infinity and $c \in D_{1}$

$$
\left\langle\tilde{q}_{1}+c \tilde{p}_{1}, \ldots, \tilde{q}_{n}+c \tilde{p}_{n}\right\rangle^{I_{y}}=\langle\widetilde{Q}\rangle^{I_{y}} .
$$

Proof. From (3.3), for any $y$ at infinity we have

$$
a_{i}^{y} \tilde{p}_{i}=b_{i 1}^{y} \tilde{q}_{1}+\cdots+b_{i n}^{y} \tilde{q}_{n}, \quad i=1, \ldots, n,
$$

where $a_{i}^{y}, b_{i j}^{y} \in S$ and $a_{i}^{y}(y) \neq 0, i=1, \ldots, n, j=1, \ldots, n$. Thus,

$$
\begin{gathered}
a_{1}^{y}\left(\tilde{q}_{1}+c \tilde{p}_{1}\right)=\left(a_{1}^{y}+c b_{11}^{y}\right) \tilde{q}_{1}+\cdots+c b_{1 n}^{y} \tilde{q}_{n}, \\
\vdots \\
a_{n}^{y}\left(\tilde{q}_{n}+c \tilde{p}_{n}\right)=c b_{n 1}^{y} \tilde{q}_{1}+\cdots+\left(a_{n}^{y}+c b_{n n}^{y}\right) \tilde{q}_{n},
\end{gathered}
$$


for any $c \in \mathbf{C}$. For $f \in\left\langle\tilde{q}_{1}+c \tilde{p}_{1}, \ldots, \tilde{q}_{n}+c \tilde{p}_{n}\right\rangle^{I_{y}}$ there exists an $h \in S$ such that $h(y) \neq 0$ and

$$
f h=\sum_{i=1}^{n} d_{i}\left(\tilde{q}_{i}+c \tilde{p}_{i}\right)
$$

where $d_{i} \in S, i=1, \ldots, n$. Multiplying both sides of (3.6) by $a_{1}^{y} \times \cdots \times a_{n}^{y}$ and using (3.5), we have $f \in\langle\widetilde{Q}\rangle^{I_{y}}$. Hence,

$$
\left\langle\tilde{q}_{1}+c \tilde{p}_{1}, \ldots, \tilde{q}_{n}+c \tilde{p}_{n}\right\rangle^{I_{y}} \subseteq\langle\widetilde{Q}\rangle^{I_{y}}
$$

for any $c \in \mathbf{C}$. For the reverse inclusion, we let

$$
A_{y}(c, z)=\left[\begin{array}{ccc}
a_{1}^{y}(z)+c b_{11}^{y}(z) & \cdots & c b_{1 n}^{y}(z) \\
c b_{21}^{y}(z) & \cdots & c b_{2 n}^{y}(z) \\
\vdots & & \vdots \\
c b_{n 1}^{y}(z) & \cdots & a_{n}^{y}(z)+c b_{n n}^{y}(z)
\end{array}\right] ;
$$

then (3.5) can be written as

$$
\left[\begin{array}{c}
a_{1}^{y}\left(\tilde{q}_{1}+c \tilde{p}_{1}\right) \\
\vdots \\
a_{n}^{y}\left(\tilde{q}_{n}+c \tilde{p}_{n}\right)
\end{array}\right]=A_{y}(c, z)\left[\begin{array}{c}
\tilde{q}_{1} \\
\vdots \\
\tilde{q}_{n}
\end{array}\right] .
$$

Let $B_{y}(c, z)$ be the determinant of $A_{y}(c, z)$ and $\bar{A}_{\underline{y}}(c, z)$ be the adjoint matrix of $A_{y}(c, z)$. Multiplying both sides of (3.8) by $\bar{A}_{y}(c, z)$ yields

$$
\bar{A}_{y}(c, z)\left[\begin{array}{c}
a_{1}^{y}\left(\tilde{q}_{1}+c \tilde{p}_{1}\right) \\
\vdots \\
a_{n}^{y}\left(\tilde{q}_{n}+c \tilde{p}_{n}\right)
\end{array}\right]=B_{y}(c, z)\left[\begin{array}{c}
\tilde{q}_{1} \\
\vdots \\
\tilde{q}_{n}
\end{array}\right] .
$$

Consider $B_{y}(c, z)$ as a polynomial in $\mathbf{C} \times M$. Denote its homogenization with respect to $c$ in $\mathbf{P}^{1} \times M$ by $\widetilde{B}_{y}\left(c_{0}, c, z\right)$. Let $B$ be the ideal generated by the $\widetilde{B}_{y}$ 's. Its zero set at infinity, denoted by $v$, is an algebraic set. Let $\pi_{1}: \mathbf{P}^{1} \times M \rightarrow \mathbf{P}^{1}$ be the natural projection. By the proper mapping theorem $\left[5\right.$, p. 64], $\pi_{1}(v)$ is an algebraic set in $\mathbf{P}^{1}$. The only algebraic subsets in $\mathbf{P}^{1}$ are the empty set, the finite-element subsets, and $\mathbf{P}^{1}$ itself. Since $\widetilde{B}_{y}(1,0, y) \neq 0$ for any $y$ at infinity, $(1,0) \notin \pi_{1}(v)$. So $\pi_{1}(v)$ is a proper algebraic set of $\mathbf{P}^{1}$ and hence is a finite set $\left\{\left(c_{i}, d_{i}\right), i=1, \ldots, k\right\}$. Let $F_{1}=\left\{\theta_{i}=\right.$ $\left.\arg \left(d_{i} / c_{i}\right) \mid c_{i} \neq 0\right\}$ and $D_{1}=\left\{r e^{i \theta} \in \mathbf{C} \mid r>0, \theta \in[0,2 \pi) \backslash F_{1}\right\}$. Then for any $y$ at infinity and $c \in D_{1}$, we have $(1, c, y) \notin v$, that is, there exists a $b \in B$ such that $b(1, c, y) \neq 0$. Since $b \in B$, we have $b=g_{1} \widetilde{B}_{y_{1}}+\cdots+g_{s} \widetilde{B}_{y_{s}}$, where $y_{1}, \ldots, y_{s}$ are some points at infinity and $g_{1}, \ldots, g_{s}$ are polynomials. From (3.9) we see that $b \tilde{q}_{i} \in\left\langle\tilde{q}_{1}+c \tilde{p}_{1}, \ldots, \tilde{q}_{n}+c \tilde{p}_{n}\right\rangle, i=1, \ldots, n$. Hence, $\langle\widetilde{Q}\rangle \subseteq\left\langle\tilde{q}_{1}+c \tilde{p}_{1}, \ldots, \tilde{q}_{n}+c \tilde{p}_{n}\right\rangle^{I_{\nu}}$, and we conclude that, by Remark 2.2,

$$
\langle\widetilde{Q}\rangle^{I_{y}} \subseteq\left\langle\tilde{q}_{1}+c \tilde{p}_{1}, \ldots, \tilde{q}_{n}+c \tilde{p}_{n}\right\rangle^{I_{y}} .
$$

This completes our proof. 
Under the same assumption of Lemma 3.2, we have the following three corollaries:

Corollary 3.1. For fixed nonzero $a$, with $a^{-1} \in D_{1}$ and any $y$ at infinity,

$$
\langle\tilde{H}(a, z, t)\rangle^{I_{y}}=\langle\widetilde{Q}\rangle^{I_{y}} \quad \text { for all } t \in[0,1) .
$$

Proof. From (3.2),

$$
\widetilde{H}(a, z, t)=(1-t) a \widetilde{Q}(z)+t \widetilde{P}(z)=(1-t) a\left(\widetilde{Q}(z)+\frac{t \widetilde{P}(z)}{(1-t) a}\right) .
$$

Since $a^{-1} \in D_{1}$, we have $t /(1-t) a \in D_{1}$ for $t \neq 1$. The assertion follows.

Corollary 3.2. For fixed $a^{-1} \in D_{1}, t \in[0,1)$, and $y$ at infinity, we have

(1) The quotient rings $S /\langle\widetilde{H}(a, z, t)\rangle$ and $S /\langle\widetilde{Q}\rangle$ have the same localization at the maximal ideal $I_{y}=\{f \in S \mid f(y)=0\}$. That is,

$$
\langle S /\langle\widetilde{H}(a, z, t)\rangle\rangle_{\left(I_{y}\right)}=\langle S /\langle\widetilde{Q}\rangle\rangle_{\left(I_{y}\right)} .
$$

Here, $I_{y}$ is considered as the maximal ideal in $S /\langle\widetilde{H}(a, z, t)\rangle$ and $S /\langle\tilde{Q}\rangle$ through canonical projections.

(2) For any prime ideal $q$ of the ring $S$, considered as the prime ideal in $S /\langle\widetilde{H}(a, z, t)\rangle$ and $S /\langle\widetilde{Q}\rangle$, with zero set $V(q)$ lying at infinity, we have

$$
\langle S /\langle\widetilde{H}(a, z, t)\rangle\rangle_{(q)}=\langle S /\langle\widetilde{Q}\rangle\rangle_{(q)} .
$$

Proof. (1) For any $f \in\langle S /\langle\widetilde{Q}\rangle\rangle_{\left(I_{y}\right)}$, we have $f=(a+q) / b$, where $q \in\langle\widetilde{Q}\rangle$ and $b(y) \neq 0$. From Corollary 3.1, $\langle\widetilde{H}(\underset{\widetilde{a}}{,}, z, t)\rangle^{I_{y}}=\langle\widetilde{Q}\rangle^{I_{y}} \supseteq\langle\widetilde{Q}\rangle$, so there exists $r \in S$ such that $r(y) \neq 0$ and $r q \in\langle\widetilde{H}(a, z, t)\rangle$. Thus,

$$
f=\frac{r(a+q)}{r b}=\frac{(r a+r q)}{r b} \in\langle S /\langle\widetilde{H}(a, z, t)\rangle\rangle_{\left(I_{y}\right)},
$$

and hence, $\langle S /\langle\tilde{H}(a, z, t)\rangle\rangle_{\left(I_{y}\right)} \supseteq\langle S /\langle\widetilde{Q}\rangle\rangle_{\left(I_{y}\right)}$. The reverse inclusion follows by the same reasoning.

(2) Let $y \in V(q)$; then $I_{y} \supset q$, and

$$
\begin{aligned}
\langle S /\langle\widetilde{H}(a, z, t)\rangle\rangle_{(q)} & =\left\langle\langle S /\langle\widetilde{H}(a, z, t)\rangle\rangle_{\left(I_{y}\right)}\right\rangle_{(q)} \\
& =\left\langle\langle S /\langle\widetilde{Q}\rangle\rangle_{\left(I_{y}\right)}\right\rangle_{(q)}=\langle S /\langle\widetilde{Q}\rangle\rangle_{(q)} .
\end{aligned}
$$

Corollary 3.3. For $a^{-1} \in D_{1}$, the intersection schemes of

$$
\bigcap_{i=1}^{n} \tilde{h}_{i}(a, z, t)
$$

at infinity are the same closed subscheme of the projective scheme $P^{k_{1}} \times \cdots \times P^{k_{m}}$ for all $t \in[0,1)$.

Proof. This follows from Corollary 3.2 and the local property of a scheme. 
Lemma 3.3. Let $P$ and $Q$ satisfy conditions (1) and (2) of Theorem 2.1, and let

$$
\bar{H}\left(\lambda_{0}, \lambda, z\right)=\lambda_{0} \widetilde{Q}+\lambda_{1} \widetilde{P}
$$

with $\left(\lambda_{0}, \lambda_{1}\right) \in \mathbf{P}^{1}$. Then for each $k$, the irreducible component $A_{k}$ of $\bar{H}^{-1}(0)$ passing through $x^{k}$ satisfies the following.

(1) Let $N$ be the set of points $\left(\lambda_{0}, \lambda_{1}, z\right)$ with $z$ at infinity; then $\pi_{1}\left(A_{k} \cap N\right) \in \mathbf{P}^{1}$ is a finite set, where $\pi_{1}$ is the natural projection;

(2) $(1,0) \notin \pi_{1}\left(A_{k} \cap N\right)$.

Proof. (1) By exercise II.3.12 of [7], $\operatorname{dim} A_{k}=1$, since $x^{k}$ is a nonsingular point of $A_{k}$. Let $B_{j}$ be any irreducible component of $A_{k} \cap N$. Since $B_{j} \neq A_{k}$, by Theorem 2, X.5, of [8], $\operatorname{dim} B_{j}<1$. So (1) follows.

(2) From the proof of Lemma 3.2, there exists a set $\bar{D}=\{\mathbf{C} \backslash$ a finite set $\}$ such that for $\lambda_{0}=1$ and $\lambda_{1} \in \bar{D}$ the intersection schemes of $\bar{H}\left(1, \lambda_{1}, z\right)$ at infinity are the same. By Proposition 9.1.2 and Example 9.1.10 of [6], for $\lambda_{1} \in \bar{D}$ and $\lambda_{0}=1$, the number of solutions of (3.10) in affine space is the same $(=r)$. Let $\varepsilon_{1}$ be small enough such that $0<\left|\lambda_{1}\right|<\varepsilon_{1}$ implies $\lambda_{1} \in \bar{D}$.

Since $\left(1,0, x^{k}\right), k=1, \ldots, r$, are nonsingular, there exists $0<\varepsilon<\varepsilon_{1}$ such that for each $0<\left|\lambda_{1}\right|<\varepsilon, \widetilde{Q}+\lambda_{1} \widetilde{P}=0$ has $r$ isolated affine solutions $x^{k}\left(\lambda_{1}\right)$ and

$$
V\left(\widetilde{Q}+\lambda_{1} \widetilde{P}\right) \cap N \cap A_{k}=\varnothing .
$$

Since $A_{k}$ is connected, (3.11) implies

$$
V(\widetilde{Q}) \cap N \cap A_{k}=\varnothing .
$$

This completes the proof.

Proof of Theorem 2.1. Le $\bar{H}(\lambda, z)=\lambda_{0} \widetilde{Q}(z)+\lambda_{1} \widetilde{P}(z)$ with $\lambda=\left(\lambda_{0}, \lambda\right) \in \mathbf{P}^{1}$. A point $(\lambda, z)$ in $\mathbf{P} \times M$ is said to be regular if and only if $\operatorname{rank} \bar{H}_{z}(\lambda, z)=n$. For each $x^{k}, k=1, \ldots, r$, in $T$, let $A_{k}$ be the irreducible component of $V(\bar{H})$, the zero set of $\bar{H}$ in $\mathbf{P}^{1} \times M$, passing through $x^{k}$. Let $B_{k}$ be the set of points in $A_{k}$ which are nonregular. Nonregularity can be described in terms of vanishing subdeterminants of $\bar{H}_{z}(\lambda, z)$, which lead to a system of polynomial equations. Consequently, $B_{k}$ is an algebraic set for each $k$. So $\pi_{1}\left(B_{k}\right)$ is a proper algebraic set in $\mathbf{P}^{1}$, because $(1,0) \notin \pi_{1}\left(B_{k}\right)$ by Lemma 3.3, and hence it is finite for each $k$. Let $A=\bigcup_{k=1}^{r} \pi_{1}\left(B_{k}\right)=\left\{\left(c_{i}^{\prime}, d_{i}^{\prime}\right) \mid i=1, \ldots, I\right\}$, $F_{2}=\left\{\theta_{i}=\arg \left(d_{i}^{\prime} / c_{i}^{\prime}\right), i=1, \ldots, I \mid c_{i} \neq 0\right\}$, and $D_{2}=\left\{r e^{i \theta} \in \mathbf{C} \mid r>0\right.$, $\left.\theta \in[0,2 \pi) \backslash F_{2}\right\}$. For $a \in \mathbf{C}$ with $a^{-1} \in D_{2}$, we have $t /(1-t) a \in D_{2}$ for all $t \in[0,1)$. That is, $(1, t /(1-t) a) \notin A$, so, $\bar{H}_{z}(1, t /(1-t) a, z)$ is of rank $n$ for any $(1, t /(1-t) a, z)$ with $t \in(0,1)$ and $z \in V(\bar{H})$. Repeated application 
of the Implicit Function Theorem on the affine representation of the homotopy

$$
0=\widetilde{H}(a, z, t)=(1-t) a \widetilde{Q}(z)+t \widetilde{P}(z)=(1-t) a \bar{H}\left(1, \frac{t}{(1-t) a}, z\right)
$$

implies the smoothness property.

For accessibility, it follows from Corollary 3.3 that for fixed $a^{-1} \in D_{1}$, the intersection schemes of $\widetilde{H}(a, z, t)$ at infinity are the same for all $t \in[0,1)$. By Proposition 9.1.2 and Example 9.1.10 of [6], for each $t$ in $[0,1)$ the number of solutions of (3.10) in affine space is the same $(=r)$. As a consequence, the $x^{k}(t)$ 's are the only solutions in affine space for each $t \in[0,1)$. By a degree theory argument as in [3], or an algebraic argument as in [7], the accessibility property follows.

By choosing $D=D_{1} \cap D_{2}$, the proof of the theorem is completed.

Proof of Proposition 2.1. For $f \in\langle\widetilde{P}\rangle^{I_{2}}$ there exists an $h \in S$ such that $h(z) \neq 0$ and $f h \in\langle\widetilde{P}\rangle$. From condition (b), $f h$ vanishes on the set of zeros of $\langle\widetilde{Q}\rangle$ at infinity. Let $x^{i}=\left(x_{1}^{i}, \ldots, x_{n}^{i}\right), i=1, \ldots, r$, be the isolated zeros of $Q$ in $\mathbf{C}^{n}$, and

$$
F(x)=\prod_{j=1}^{r} \sum_{i=1}^{n} e_{i}\left(x_{i}-x_{i}^{j}\right),
$$

where $e_{i} \in \mathbf{C}, i=1, \ldots, n$, are chosen such that $\widetilde{F}(z) \neq 0$. It is easy to see that $\widetilde{F}\left(z^{i}\right)=0$ for each $i=1, \ldots, r$, where $z^{i}$ is the corresponding point of $x^{i}$ in $M$, and $\tilde{F} h f$ vanishes on $V(\widetilde{Q})$. By the Nullstellensatz, $(\widetilde{F} h f)^{k} \in\langle\widetilde{Q}\rangle$ for some positive integer $k$. Since $(\widetilde{F} h)^{k}(z) \neq 0$, we have $f^{k} \in\langle\widetilde{Q}\rangle^{I_{z}}$. By Theorem 48 of [13], $\langle\widetilde{Q}\rangle^{I_{z}}$ is a prime ideal. Hence, $f \in\langle\widetilde{Q}\rangle^{I_{z}}$, which completes our proof.

Proof of Proposition 2.2. $(\Rightarrow)$ For any $p$ at infinity, the $F$ chosen in (3.14) gives $\widetilde{F}(p) \neq 0$, and $\widetilde{F} x_{0}$ vanishes on $V(\widetilde{Q})$. By the Nullstellensatz, $\left(x_{0}\right)^{k} \in\langle\widetilde{Q}\rangle^{I_{p}}$. Now, for $h \in\langle\widetilde{P}\rangle^{I_{p}}$ there exist $g \in S=\mathbf{C}\left[x_{0}, \ldots, x_{n}\right]$ and $x_{l}$ such that $g(p) \neq 0, x_{l}(p) \neq 0$, and

$$
h g=\sum_{i=1}^{n} a_{i} \tilde{p}_{i},
$$

where $a_{i} \in S, i=1, \ldots, n$. Without loss of generality, we may assume $h g$ is homogeneous of degree, say, $s$. Then, for large enough $e$ and $k,(h g) x_{l}^{j} \in$ $\langle\widetilde{P}\rangle_{e} \subset\left\langle\widetilde{Q}, x_{0}^{k}\right\rangle_{e}=\langle\widetilde{Q}\rangle_{e}$, where $j=e-s$. Hence, $h \in\langle\widetilde{Q}\rangle^{I_{p}}$.

$(\Leftrightarrow)$ For $f \in S$ and $I$ an ideal of $S$, let

$$
R(f, I)=\{g \in S \mid f g \in I\} .
$$

If the zero set $V(R(f, I))$ of $R(f, I)$ is empty, then the polynomials $x_{i}, i=$ $0, \ldots, n$, vanish at every point of $V(R(f, I))$. By the Nullstellensatz, $\left(x_{j}\right)^{a} \in$ $R(f, I)$ for $a \gg 1$. Hence, when $e$ is large enough, the set of homogeneous 
polynomials of degree $e$ in $R(f, I)$, denoted by $R(f, I)_{e}$, equals $S_{e}$, the set of homogeneous polynomials of degree $e$ in $S$. That is,

$$
R(f, I)_{e}=S_{e} .
$$

For fixed $k$, let $f \in\left\langle\widetilde{P}, x_{0}^{k}\right\rangle_{e}$. To prove $f \in\left\langle\tilde{Q}, x_{0}^{k}\right\rangle_{e}$ for large enough $e$, from (3.15), it suffices to show $V=V\left(R\left(f,\left\langle\widetilde{Q}, x_{0}^{k}\right\rangle\right)\right)=\varnothing$. It is clear that $x_{0}^{k} \in R\left(f,\left\langle\widetilde{Q}, x_{0}^{k}\right\rangle\right)$, so $V$ is supported at infinity. But for any $p$ at infinity, (2.4) implies that there exists $h \in S, h(p) \neq 0$, and $f h \in\langle\widetilde{Q}\rangle$. Hence, $h \in R\left(f,\left\langle\widetilde{Q}, x_{0}^{k}\right\rangle\right)$ and $p \notin V$. This completes the proof.

\section{APPENDIX}

For $P=\left(p_{1}, p_{2}\right)$ in (1.5) and $Q=\left(q_{1}, q_{2}\right)$ in (1.6), let $H=\left(h_{1}, h_{2}\right)=$ $(1-t) a Q+t P$, where $a$ is any nonzero number in $\mathbf{C}$ which is not a negative real number. To be precise,

$$
\begin{gathered}
h_{1}\left(a, x_{1}, x_{2}, t\right)=(1-t) a\left(x_{2}^{2}-1\right)+t\left(x_{2}^{2}+x_{1}\right)=0, \\
h_{2}\left(a, x_{1}, x_{2}, t\right)=(1-t) a\left(x_{2}^{2}+x_{1} x_{2}\right)+t\left(x_{2}^{2}+x_{2}\right)=0 .
\end{gathered}
$$

Multiplying (1) by $x_{2}(1-t) a$ and subtracting $t \times(2)$ yields

(3) $x_{2}^{3}\left[(1-t) a t+(1-t)^{2} a^{2}\right]+x_{2}^{2}\left[-(1-t) a t-t^{2}\right]+x_{2}\left[-(1-t)^{2} a^{2}-t^{2}\right]=0$.

From (3), we can see that for each fixed $a \in \mathbf{C}$ and $t \in(0,1)$, the zero set of $H\left(a, x_{1}, x_{2}, t\right)$ is $\left(x_{1}, x_{2}\right)=((1-t) a / t, 0),\left(d_{1}, e_{1}\right)$, and $\left(d_{2}, e_{2}\right)$, where

$$
\begin{gathered}
e_{1}=\frac{-b+\sqrt{b^{2}-4 c}}{2}, \quad e_{2}=\frac{-b-\sqrt{b^{2}-4 c}}{2}, \\
d_{i}=-\frac{t\left(e_{i}+1\right)}{(1-t) a}-e_{i}, \quad i=1,2,
\end{gathered}
$$

or

$$
d_{i}=-\frac{\left[\left(\left(e_{i}\right)^{2}-1\right)(1-t) a-\left(e_{i}\right)^{2} t\right]}{t}, \quad i=1,2,
$$

with

$$
b=\frac{-t}{(1-t) a}, \quad c=\frac{-(1-t)^{2} a^{2}-t^{2}}{(1-t) a[t+(1-t) a]} .
$$

It is easy to see that as $t \rightarrow 0$, we have $b \rightarrow 0, c \rightarrow-1$. Hence, from (4) and (5), $\left(d_{1}, e_{1}\right) \rightarrow(-1,1)$ and $\left(d_{2}, e_{2}\right) \rightarrow(1,-1)$. When $t \rightarrow 1$, then $\frac{4 c}{b^{2}} \rightarrow 0, \frac{c}{b}$ is bounded and

$$
\begin{aligned}
f & \equiv-b+\sqrt{b^{2}-4 c}=b\left(-1+\sqrt{1-\frac{4 c}{b^{2}}}\right) \\
& =b\left(-1+1-\frac{2 c}{b^{2}}+o\left(\frac{4 c}{b^{2}}\right)\right)=\frac{-2 c}{b}+o\left(\frac{4 c}{b}\right) .
\end{aligned}
$$


However,

$$
\frac{c}{b}=\frac{\left[t^{2}+(1-t)^{2} a^{2}\right]}{t[t+(1-t) a]} \rightarrow 1 \quad \text { as } t \rightarrow 1 \text {. }
$$

Hence, $f \rightarrow-2$ and $e_{1} \rightarrow-1$ as $t \rightarrow 1$. From $(6),\left(d_{1}, e_{1}\right) \rightarrow(-1,-1)$. Similarly,

$$
\begin{aligned}
g & \equiv-b-\sqrt{b^{2}-4 c}=b\left(-1-\sqrt{1-\frac{4 c}{b^{2}}}\right) \\
& =b\left(-1-\left[1-\frac{2 c}{b^{2}}+o\left(\frac{4 c}{b^{2}}\right)\right]\right) \\
& =-2 b+\frac{2 c}{b}+o\left(\frac{4 c}{b^{2}}\right) .
\end{aligned}
$$

When $t \rightarrow 1$, then $b \rightarrow+\infty$, hence, $g \rightarrow+\infty$ and $e_{2} \rightarrow+\infty$. Therefore, $\left(d_{2}, e_{2}\right) \rightarrow(+\infty,+\infty)$ from $(6)$.

\section{BIBLIOGRAPHY}

1. E. Allgower and K. Georg, Simplicial and continuation methods for approximating fixed points and solutions to systems of equations, SIAM Rev. 22 (1980), 22-85.

2. V. Balakotaiah, D. Luss, and B. L. Keyfitz, Steady state multiplicity analysis of lumpedparameter systems described by a set of algebraic equations, Chem. Engrg. Comm. 36 (1985), 121-147.

3. S.-N. Chow, J. Mallet-Paret, and J. A. Yorke, A homotopy method for locating all zeros of a system of polynomials, Functional Differential Equations and Approximation of Fixed Points (H.-D. Peitgen and H.-O. Walther, eds.), Lecture Notes in Math., vol. 730, Springer, New York, 1979, pp. 77-88.

4. M. Chu, T. Y. Li, and T. Sauer, A homotopy method for general $\lambda$-matrix problems, SIAM J. Matrix. Anal. Appl. 9 (1988), 528-536.

5. G. Fischer, Complex analytic geometry, Lecture Notes in Math., vol. 538, Springer, New York, 1976.

6. W. Fulton, Intersection theory, Springer, New York, 1984.

7. R. Hartshorne, Algebraic geometry, Graduate Texts in Math., vol. 52, Springer, Berlin, Heidelberg, New York, 1977.

8. W. V. D. Hodge and D. Pedoe, Methods of algebraic geometry, Vol. 2, Cambridge Univ. Press, 1952.

9. T. Y. Li, On the Chow, Mallet-Paret and Yorke homotopy for solving systems of polynomials, Bull. Inst. Math. Acad. Sinica 11 (1983), 433-437.

10. T. Y. Li and T. Sauer, A simple homotopy for solving deficient polynomial systems, Japan J. Appl. Math. 6 (1989), 11-21.

11. T. Y. Li, T. Sauer, and J. A. Yorke, Numerical solution of a class of deficient polynomial systems, SIAM J. Numer. Anal. 24 (1987), 435-451.

12. _ The random product homotopy and deficient polynomial systems, Numer. Math. 51 (1987), 481-500.

13. H. Matsumura, Commutative algebra, Benjamin, New York, 1970.

14. A. Morgan, A homotopy for solving polynomial systems, Appl. Math. Comput. 18 (1986), 86-92. 
15. A. Morgan and A. Sommese, A homotopy for solving general polynomial systems that respects m-homogeneous structures, Appl. Math. Comput. 24 (1987), 101-113.

16. Computing all solutions to polynomial systems using homotopy continuation, Appl. Math. Comput. 24 (1987), 115-138.

17. W. Zulehner, A simple homotopy method for determining all isolated solutions to polynomial systems, Math. Comp. 50 (1988), 167-177.

Department of Mathematics, Michigan State University, East Lansing, Michigan 48824-1027

E-mail address: li@nsf1.mth.msu.edu

Permanent address, X. Wang: Department of Mathematics, Jilin University, Changchun, People's Republic of China 